\title{
Baryogenesis through mixing of heavy Majorana neutrinos
}

\section{Apostolos Pilaftsis}

Theory Division, CERN, CH-1211 Geneva 23, Switzerland

E-mail: pilaftsi@mail. cern. chi

Abstract: We review the scenario of baryogenesis through leptogenesis induced by the out-of-equilibrium decays of heavy neutrinos. We pay special attention to the resonant phenomenon of $\mathrm{CP}$ violation through mixing of two nearly degenerate heavy Majorana neutrinos and show how unitarity and CPT invariance is maintained within the resummation approach. An important consequence of this is that the leptogenesis scale may be as low as $1 \mathrm{TeV}$, even for models with universal Yukawa couplings. We briefly discuss the impact of finite temperature effects and low-energy constraints to the afore-mentioned mechanism of $\mathrm{CP}$ violation.

\section{Introduction}

Many scenarios have been invoked in order to explain the small baryon-to-photon ratio of number densities $n_{B} / n_{\gamma}=(4-7) \times 10^{-10}$, which is found by present observations. Among them, the most attractive scenario is the one proposed by Fukugita and Yanagida [in'] which may lead to a consistent solution to the problem of the baryon asymmetry in the Universe (BAU). In their model, out-of-equilibrium $L$-violating decays of heavy Majorana neutrinos $N_{i}$ with masses $m_{N i}$ much larger the critical temperature $T_{c} \approx$ $200 \mathrm{GeV}$ produce an excess in the lepton number $L$. Then, the excess in $L$ is converted into the desired $B$ asymmetry through $B+L$-violating sphaleron interactions [2], which are in thermal equilibrium for temperatures ranging from $T_{c}$ up

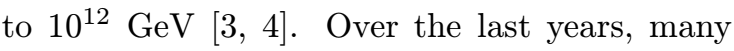
studies have been devoted to this mechanism of

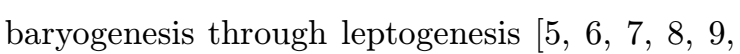
[1].'.

In $[\overline{1} i$, the necessary $\mathrm{CP}$ violation in heavy Majorana neutrino decays results from the interference between the tree-level graph and the absorptive part of the one-loop vertex. Using the known terminology of the $K^{0} \bar{K}^{0}$ system [i] $\overline{1}_{1}^{1}$, we call this mechanism of $\mathrm{CP}$ violation as $\varepsilon^{\prime}$-type $\mathrm{CP}$ violation. The $\varepsilon^{\prime}$-type $\mathrm{CP}$ violation was dis-

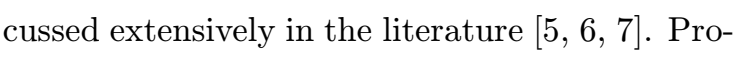
vided all Yukawa couplings of the Higgs fields to $N_{i}$ and lepton isodoublets $L$ are of comparable order $\left[\begin{array}{l}1 \\ {[1}\end{array}\right.$ mechanism requires very heavy Majorana neutrinos of order $10^{7}-10^{8} \mathrm{GeV}$. This high mass bound may be lifted if a strong hierarchy for the Yukawa couplings and the heavy Majorana neu-

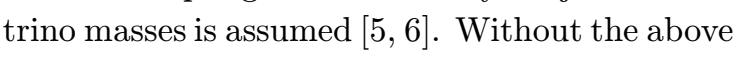
assumption on the Yukawa couplings however [i], one finds that $\varepsilon^{\prime}<10^{-15}$ for $m_{N i} \approx 1 \mathrm{TeV}$, which is far too small to account for the BAU.

In heavy particle decays responsible for the baryon (lepton) asymmetry, CP violation may also arise from the interference of the tree-level graph with the absorptive part of a self-energy transition describing the mixing of the decaying

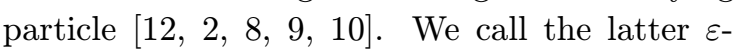
type CP violation. Recently, it has been observed in $\left[\overline{9}_{1}^{1}, 10\right]$, that $\mathrm{CP}$ violation can be considerably enhanced through the mixing of two nearly degenerate heavy Majorana neutrinos. A minimal model with nearly degenerate neutrinos may be the low-energy limit of $\mathrm{E}_{6}$ or $\mathrm{SO}(10)$ theories. We will discuss such a scenario in Section 2.

In order to describe the dynamics of $\mathrm{CP}$ violation through unstable particle mixing, one is compelled to rely on resummation approaches, 
which treat unstable particles in a consistent way and preserve all desirable field-theoretic properties such as gauge-invariance, analyticity, CPT invariance and unitarity. In the context of gauge field theories, such a resummation approach to resonant transition amplitudes has been formulated, which is implemented by the pinch technique [1 13 ' $]$. Then, this formalism has been extended to the case of mixing between two intermediate resonant states in scattering processes

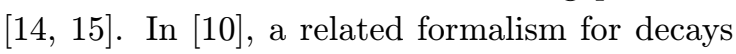
has been developed, which effectively takes into account decoherence phenomena in the mixing and subsequent decay of heavy particles, namely heavy neutrinos. Within this formalism, we have shown that $\varepsilon$-type $\mathrm{CP}$ violation can be even of order unity [10 $0_{1}^{\prime}$. Thus, we find agreement with earlier articles on resonant $\mathrm{CP}$ violation in scatterings involving top quarks, supersymmetric quarks or Higgs particles in the intermediate state $\left[1 \overline{6}_{n}^{\prime}\right.$ 114, 15!.

In Section 3, we will briefly describe the aforementioned resummation approach and show how unitarity and CPT invariance is maintained. Based on this approach, we will calculate the leptonic $\mathrm{CP}$ asymmetries of heavy neutrinos and derive the sufficient and necessary conditions for orderunity $\mathrm{CP}$ violation. As we will see, this very last fact allows one to lower the scale of leptogenesis up to TeV scales [i10"], even for models without any large Yukawa-coupling hierarchy. In Section 4, we discuss the implications of low-energy constraints such as the electric dipole moment (EDM) of electron for the leptogenesis scenario. In Section 5, we examine the impact of finitetemperature effects on the resonant mechanism of $\mathrm{CP}$ violation and give predictions of the baryonic asymmetry in two representative scenarios, after solving numerically the relevant Boltzmann equations. Section 6 contains the conclusions.

\section{Heavy Majorana neutrino models}

Heavy Majorana neutrinos may naturally be realized in certain GUT's, such as $\mathrm{SO}(10)$ [ [1 $\overline{1} \overline{7}_{\mathrm{b}}^{\prime}, \overline{1} \overline{8}_{1}^{\prime}$ and/or $\mathrm{E}_{6}\left[1 \mathrm{1}_{1}^{1}\right]$ models. Nevertheless, these models will also predict several other particles, e.g., leptoquarks, additional charged and neutral gauge bosons $\left(W_{R}^{ \pm}\right.$and $Z_{R}$ ), which may deplete the number density of heavy neutrinos $N_{i}$ through processes of the type $N_{i} \bar{e}_{R} \rightarrow W^{+*} \rightarrow u_{R} \bar{d}_{R}$ and so render the whole analysis very involved. If these particles are sufficiently heavier than the lightest heavy Majorana neutrino and/or the temperature of the universe [i5n completely avoided. Since we wish to simplify our analysis without sacrificing any of the essential features involved in the study of the BAU, we shall consider a minimal model with isosinglet neutrinos, which is invariant under the SM gauge group, $\mathrm{SU}(2)_{L} \otimes \mathrm{U}(1)_{Y}$. To this end, we consider a minimal scenario, in which the fermionic matter of the SM is extended by adding two righthanded neutrinos per family, e.g., $\nu_{l R}$ and $\left(S_{l L}\right)^{C}$ with $l=e, \mu, \tau$. Such a scenario may be derived from certain $\mathrm{SO}(10)$ [1] $\left.\overline{1}_{1}^{\prime}\right]$ and/or $\mathrm{E}_{6}\left[{ }_{1}^{1} \overline{9}_{1}^{\prime}\right]$ models. For our illustrations, we neglect possible interfamily mixings. Imposing lepton-number conservation on the model gives rise to the Lagrangian

$$
\begin{aligned}
-\mathcal{L}= & \frac{1}{2}\left(\bar{S}_{L},\left(\bar{\nu}_{R}\right)^{C}\right)\left(\begin{array}{cc}
0 & M \\
M & 0
\end{array}\right)\left(\begin{array}{c}
\left(S_{L}\right)^{C} \\
\nu_{R}
\end{array}\right) \\
& +h_{R}\left(\bar{\nu}_{L}, \bar{l}_{L}\right) \tilde{\Phi} \nu_{R}+\text { H.c. },
\end{aligned}
$$

where $\tilde{\Phi}=i \sigma_{2} \Phi$ is the isospin conjugate Higgs doublet and $\sigma_{2}$ is the usual Pauli matrix. There are now two equivalent ways to break both $L$ and CP invariance of the Lagrangian in Eq. (2.1): One has to either (i) introduce two complex $L$ violating mass terms of the kind $\mu_{R} \bar{\nu}_{R} \nu_{R}^{C}$ and $\mu_{L} \bar{S}_{L}^{C} S_{L}$, where both $\mu_{R}$ and $\mu_{L}$ are complex, or equivalently (ii) add the $L$-violating coupling $h_{L}\left(\bar{\nu}_{L}, \bar{l}_{L}\right) \tilde{\Phi}\left(S_{L}\right)^{C}$ and include one $L$-violating mass parameter, e.g., $\mu_{R} \bar{\nu}_{R} \nu_{R}^{C}$. The origin of these parameters are usually due to residual effects of high-dimensional operators involving superheavy neutrinos [1 19$]$. The typical size of the $L$ violating parameters is $\mu_{L}, \mu_{R} \sim M^{2} / M_{\text {Planck }}$, $M^{2} / M_{X}$ or $M^{2} / M_{S}$, where $M_{S} \approx 10^{-3} M_{X}$ is some intermediate see-saw scale. As a consequence, such effective minimal models derived from $\mathrm{E}_{6}$ theories can naturally predict small mass splittings for the heavy neutrinos $N_{1}$ and $N_{2}$. To a good approximation, this small mass difference may be determined from the parameter $x_{N}=m_{N_{2}} / m_{N_{1}}-1 \sim \mu_{L} / M$ or $\mu_{R} / M$. For instance, if $M=10 \mathrm{TeV}$ and $\mu_{L}=\mu_{R}=M^{2} / M_{X}$, one then finds $x_{N} \approx 10^{-12}-10^{-11}$. As we will 
see in Section 4, these small values for the mass difference $x_{N}$ can produce large CP asymmetries in the heavy neutrino decays.

Using generalized CP transformations [201], one can derive the sufficient and necessary condition for CP invariance in a general weak basis of the model. The condition is given by [10

$$
\begin{aligned}
& \Im m \operatorname{Tr}\left(h^{\dagger} h M^{\nu \dagger} M^{\nu} M^{\nu \dagger} h^{T} h^{*} M^{\nu}\right)= \\
& m_{N_{1}} m_{N_{2}}\left(m_{N_{1}}^{2}-m_{N_{2}}^{2}\right) \Im m\left(h_{l 1} h_{l 2}^{*}\right)^{2}=0 .
\end{aligned}
$$

where $h=\left(h_{l 1}, h_{l 2}\right)$ is a $1 \times 2$ Yukawa matrix defined in the mass basis, i.e., $M^{\nu}=\widehat{M}^{\nu}$. From Eq. $(2.21)$, one readily sees that only one physical CP-violating combination is possible in this minimal model and $\mathrm{CP}$ invariance is restored if $m_{N_{1}}=m_{N_{2}}$ provided none of the isosinglet neutrinos is massless. The above considerations may be extended to models with more than two righthanded neutrinos and more than one lepton families. In this case, there may be more conditions analogous to Eq. (2.21), which involve high order terms in the Yukawa-coupling matrix $h$. However, not all of the conditions are sufficient and necessary for CP invariance. Instead of undertaking the rather difficult task to derive all CPinvariant conditions, we note in passing that the total number $\mathcal{N}_{C P}$ of all non-trivial CP-violating phases in a model with $n_{L}$ weak isodoublets and $n_{R}$ neutral isosinglets is $\mathcal{N}_{C P}=n_{L}\left(n_{R}-1\right)$ [2 $\left.{ }^{\prime} 1_{1}^{\prime}\right]$.

\section{Resummation approach: CP asymmetries and CPT invariance}

Here, our main concern is to present an approach to decay amplitudes that describes the dynamics of unstable particle mixing. Because of the many decoherent collisions of the heavy particle with the thermal bath in the early universe [222], we must take into account only the incoherent part of the mixing and decay of the unstable particle. This will be done in an effective manner, such that the incoherent decay amplitude derived with this method can be embedded in an equivalent form to a transition element $\left[13_{1}^{1}, 1 \overline{1}=1\right]$ in agreement with Veltman's S-matrix approach [2]

Our approach is inspired by the LSZ reduction formalism [24]. Suppose that we wish to find the effective resummed amplitude $\mathcal{T}_{N_{1}}$ for

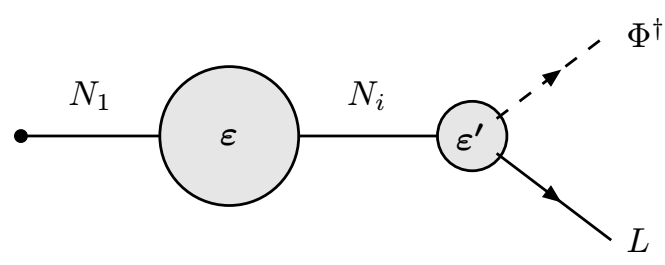

Figure 1: $\varepsilon$ - and $\varepsilon^{\prime}$-type $\mathrm{CP}$ violation in the decays of heavy Majorana neutrinos.

the decay $N_{1} \rightarrow L \Phi^{\dagger}$ which includes $N_{1} N_{2}$ mixing. Then, we start with the Green function $\Phi^{\dagger} L N_{1}$ shown in Fig. 1 and amputate the external legs by their inverse propagators. For the stable-particle lines $L$ and $\Phi$, the procedure does not differ from the usual one known from field theory. For the external line describing the $N_{1} N_{2}$ system, one has first to consider the resummed propagator of the $N_{1} N_{2}$ system

$$
\begin{aligned}
& S_{i j}(\not p)= \\
& {\left[\begin{array}{cc}
\not p-m_{N_{1}}+\Sigma_{11}(\not p) & \Sigma_{12}(\not p) \\
\Sigma_{21}(\not p) & \not p-m_{N_{2}}+\Sigma_{22}(\not p)
\end{array}\right]}
\end{aligned}
$$

where $m_{N_{1,2}}$ are the heavy neutrino masses, $\Sigma_{i j}(\not \not)$ are the $N_{j} \rightarrow N_{i}$ self-energy transitions which are renormalized in the on-shell (OS) renormalization scheme [25i]. Then, as we did for the stable-particle lines, we have to truncate the resummed $N_{i} N_{j}$ propagator in Eq. (33.1) by the inverse $N_{1} N_{1}$ propagator. To this end, we need to calculate the propagators

$$
\begin{aligned}
& S_{11}(\not p)= {\left[\not p-m_{N_{1}}+\Sigma_{11}(\not p)\right.} \\
&\left.-\Sigma_{12}(\not p) \frac{1}{\not p-m_{N_{2}}+\Sigma_{22}(\not p)} \Sigma_{21}(\not p)\right]^{-1}, \\
& S_{21}(\not p)=- {\left[\not p-m_{N_{2}}+\Sigma_{22}(\not p)\right]^{-1} } \\
& \Sigma_{21}(\not p) S_{11}(\not p) .
\end{aligned}
$$

Employing now the LSZ reduction procedure, we obtain the effective decay amplitude

$$
\begin{aligned}
\mathcal{T}_{N_{1}} & =\mathcal{T}_{N_{i}}^{a m p} S_{i 1}(\not p) S_{11}^{-1}(\not p) u_{N_{1}}(p) \\
& =\bar{u}_{l} P_{R}\left\{h_{l 1}+i \mathcal{V}_{l 1}^{a b s}(\not p)-i\left[h_{l 2}+i \mathcal{V}_{l 2}^{a b s}(\not p)\right]\right.
\end{aligned}
$$




$$
\left.\times\left[\not p-m_{N_{2}}+i \Sigma_{22}^{a b s}(\not p)\right]^{-1} \Sigma_{21}^{a b s}(\not p)\right\} u_{N_{1}}
$$

where $\mathcal{T}_{N_{i}}^{a m p}$ is the OS renormalized $\Phi^{\dagger} l N_{i}$ vertex, $\Sigma_{i j}^{a b s}(\not p)$ and $\mathcal{V}_{l i}^{a b s}(\not p)$ denote the absorptive parts of the one-loop self-energy transition $N_{j} \rightarrow N_{i}$ and the one-loop irreducible vertex $\Phi^{\dagger} l N_{i}$, respectively. The self-energy and vertex functions are given by

$$
\begin{aligned}
& \Sigma_{i j}^{a b s}(\not p)=\frac{h_{l i} h_{l j}^{*}}{16 \pi} \not p P_{L}+\frac{h_{l i}^{*} h_{l j}}{16 \pi} \not p P_{R}, \\
& \mathcal{V}_{l i}^{a b s}(\not p)=-\frac{h_{l^{\prime} i}^{*} h_{l^{\prime} j} h_{l j}}{16 \pi \sqrt{p^{2}}} \not p P_{L} f\left(\frac{m_{N j}^{2}}{p^{2}}\right),
\end{aligned}
$$

with $P_{R(L)}=\left[1+(-) \gamma_{5}\right] / 2$ and $f(x)=\sqrt{x}[1-$ $\left.(1+x) \ln (1+1 / x)][]_{1}^{1}\right]$. The CP-conjugate decay amplitude $\overline{\mathcal{T}}_{N_{1}}$ for $\bar{N}_{1} \rightarrow L^{C} \Phi$ may be obtained by taking the complex conjugate for the Yukawa couplings and replacing $P_{R}$ with $P_{L}$ in Eq. (3.4). Analogously, one can find $\mathcal{T}_{N_{2}}$ and $\overline{\mathcal{T}}_{N_{2}}$.

Our main interest is now in the physical CPviolating observables

$$
\delta_{N_{i}}=\frac{\Gamma\left(N_{i} \rightarrow L \Phi^{\dagger}\right)-\Gamma\left(N_{i} \rightarrow L^{C} \Phi\right)}{\Gamma\left(N_{i} \rightarrow L \Phi^{\dagger}\right)+\Gamma\left(N_{i} \rightarrow L^{C} \Phi\right)},
$$

for $i=1,2$. Since we wish to study the dominant mechanisms of $\mathrm{CP}$ violation in the heavy neutrino decays, we can define the $\mathrm{CP}$-violating quantities $\varepsilon_{N_{i}}$ and $\varepsilon_{N_{i}}^{\prime}$ analogous to Eq. (13.6) by considering only the self-energy or only the vertex contribution to $\delta_{N_{i}}$. Then, for the vertex contribution to CP asymmetries, we find the known result [i]1, [1]

$$
\varepsilon_{N_{1}}^{\prime}=\frac{\Im m\left(h_{l 1}^{*} h_{l 2}\right)^{2}}{8 \pi\left|h_{l 1}\right|^{2}} f\left(\frac{m_{N_{2}}^{2}}{m_{N_{1}}^{2}}\right) .
$$

In the degenerate heavy-neutrino mass limit $\left[m_{N_{1}}=\right.$ $\left.m_{N_{2}}=m_{N}\right], \varepsilon_{N_{1}}^{\prime}$ contribution cancels against $\varepsilon_{N_{2}}^{\prime}$, so no net CP violation can be generated in agreement with the condition in Eq. (2.2).

Based on the resummation approach outlined above (c.f. Eq. $\left.\left(\underline{3} \cdot \overline{4}^{4}\right)\right)$, we can calculate the contribution which is entirely due to heavy-neutrino self-energy effects. In this way, we find the simple formula [i] ${ }^{1} 0_{1}^{\prime \prime}$

$$
\varepsilon_{N_{1}} \approx \frac{\Im m\left(h_{l 1}^{*} h_{l 2}\right)^{2}}{\left|h_{l 1}\right|^{2}\left|h_{l 2}\right|^{2}} \frac{\Delta m_{N}^{2} m_{N_{1}} \Gamma_{N_{2}}}{\left(\Delta m_{N}^{2}\right)^{2}+m_{N_{1}}^{2} \Gamma_{N_{2}}^{2}},
$$

where $\Delta m_{N}^{2}=m_{N_{1}}^{2}-m_{N_{2}}^{2}$ and $\Gamma_{N_{i}}=\left|h_{l i}\right|^{2} m_{N_{i}} /(8 \pi)$ are the decay widths of the heavy Majorana neutrinos. Equation (3.81) is a very good approximation for any range of heavy-neutrino masses of interest. An analogous expression can be obtained for $\varepsilon_{N_{2}}$ just by changing the indices from 1 to 2 and vice versa in Eq. (3.8. Both CP asymmetries $\varepsilon_{N_{1}}$ and $\varepsilon_{N_{2}}$ are of the same sign and go individually to zero when $\Delta m_{N}^{2} \rightarrow 0$, as it should be on account of Eq. (12.21). In the conventional perturbation theory, the width term $m_{N_{1}}^{2} \Gamma_{N_{2}}^{2}$ occurring in the last denominator on the RHS of Eq. (3.8) is absent, and this very last fact is precisely what causes a singular behaviour when the degeneracy between the two heavy Majorana neutrinos is exact. On physical grounds, however, this should not be very surprising, since the only natural parameter that can regulate such a singularity is the finite width of the heavy neutrinos which is naturally implemented within the
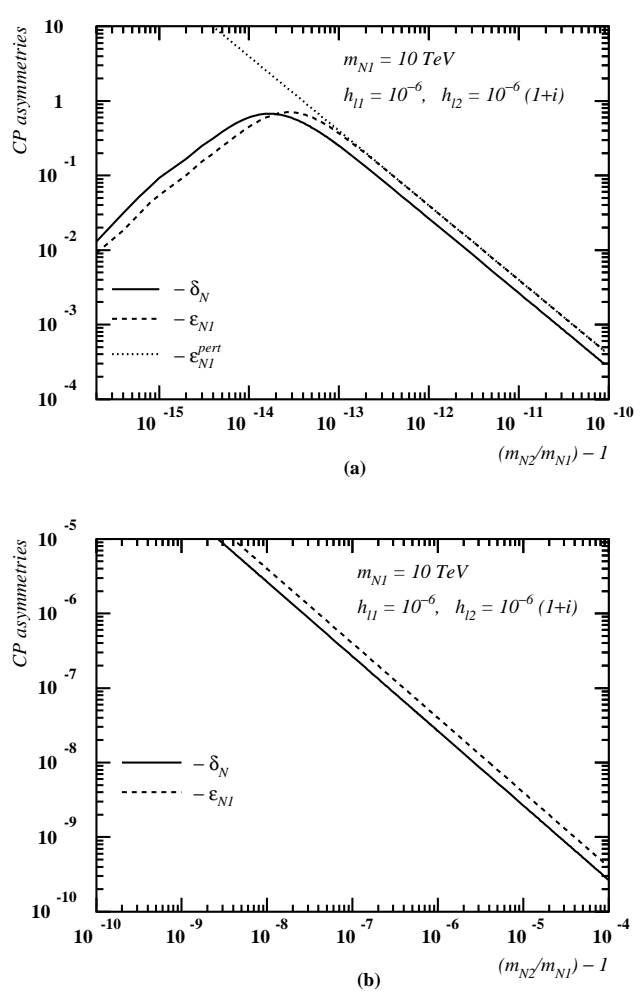

Figure 2: Dependence of CP asymmetries on $\Delta m_{N}^{2}$. 
resummation approach.

From Eq. (3.8. and necessary conditions for maximal $\mathrm{CP}$ violation. Thus, we have a resonant enhancement of $\mathrm{CP}$ violation of order unity iff

$$
\begin{aligned}
m_{N_{1}}-m_{N_{2}} \sim \pm A_{22} m_{N_{2}} & =\frac{\Gamma_{N_{2}}}{2} \\
\text { and/or } \quad A_{11} m_{N_{1}} & =\frac{\Gamma_{N_{1}}}{2} .
\end{aligned}
$$

and

$$
\delta_{C P}=\frac{\left|\Im m\left(h_{l 1}^{*} h_{l 2}\right)^{2}\right|}{\left|h_{l 1}\right|^{2}\left|h_{l 2}\right|^{2}} \approx 1 .
$$

A numerical example of resonant $\mathrm{CP}$ violation is displayed in Fig. 2, where $\varepsilon_{N_{1}}^{\text {pert }}$ is the result in the conventional perturbation theory and $\delta_{N}$ comprises the total CP-violating effect of both $N_{1}$ and $N_{2}$ decays.

It is now interesting to demonstrate how CPT invariance and unitarity is preserved within the resummation approach "1; immediate consequence of unitarity and CPT symmetry is that $\mathrm{CP}$ violation in the $L$-violating scattering process $L \Phi^{\dagger} \rightarrow L^{C} \Phi$ is absent to order $h_{l i}^{6}\left[\overline{2} \overline{6}_{1}^{1}, \overline{2}_{2} z_{1}\right]$. We will concentrate on the resonant part of the amplitude which is the dominant one. Thus, we wish to show that to 'one loop',

$$
\begin{aligned}
\Delta_{\mathrm{CP}} & =\int d \operatorname{LIPS}\left|\mathcal{T}_{L \Phi^{\dagger} \rightarrow L^{C} \Phi}^{\mathrm{res}}\right|^{2} \\
& -\int d \operatorname{LIPS}\left|\overline{\mathcal{T}}_{L^{C} \Phi \rightarrow L \Phi^{\dagger}}^{\mathrm{res}}\right|^{2}=0,
\end{aligned}
$$

where LIPS stands for the two-body Lorentz invariant phase space. Furthermore, omitting external spinors and absorbing irrelevant constants in the definition of the Yukawa-coupling matrix $h=\left(h_{l 1}, h_{l 2}\right)$, we have in matrix notation

$$
\begin{aligned}
\mathcal{T}_{L \Phi^{\dagger} \rightarrow L^{C} \Phi}^{\mathrm{res}} & =h P_{R} S(\not p) P_{R} h^{T}, \\
\overline{\mathcal{T}}_{L^{C} \Phi \rightarrow L \Phi^{\dagger}}^{\mathrm{res}} & =h^{*} P_{L} \bar{S}(\not p) P_{L} h^{\dagger},
\end{aligned}
$$

with $\bar{S}(\not p)=S^{T}(\not p)$ being the $\mathrm{CP} / \mathrm{T}$-conjugate propagator matrix of $S(\not p)$.

We start our proof by noticing first that as a consequence of CPT invariance,

$$
\left|\mathcal{T}_{L \Phi^{\dagger} \rightarrow L \Phi^{\dagger}}^{\text {res }}\right|^{2}=\left|\mathcal{T}_{L^{C} \Phi \rightarrow L^{C} \Phi}^{\text {res }}\right|^{2},
$$

since

$$
\begin{aligned}
\left|h P_{R} S(\not p) P_{L} h^{\dagger}\right| & =\left|h^{*} P_{L} S^{T}(\not p) P_{R} h^{T}\right| \\
& =\left|h^{*} P_{L} \bar{S}(\not p) P_{R} h^{T}\right|
\end{aligned}
$$

Then, based on the unitarity relation, also known as the optical theorem, governing the resummed propagators, i.e.

$$
\begin{aligned}
& S^{-1}(\not p)-S^{-1 \dagger}(\not p)= \\
& \quad-i \int d \operatorname{LIPS} \not p\left(h^{T} h^{*} P_{L}+h^{\dagger} h P_{R}\right),
\end{aligned}
$$

we can prove the equality

$$
\begin{gathered}
\int d \operatorname{LIPS}\left|\mathcal{T}_{L^{\dagger} \Phi^{\dagger} \rightarrow L \Phi^{\dagger}, L^{C} \Phi}^{\mathrm{res}}\right|^{2}= \\
\int d \operatorname{LIPS}\left|\overline{\mathcal{T}}_{L^{C} \Phi \rightarrow L \Phi^{\dagger}, L^{C} \Phi}^{\mathrm{res}}\right|^{2} .
\end{gathered}
$$

Indeed, using Eq. ('s.15), we find

$$
\begin{aligned}
\int d \operatorname{LIPS} & \left|\mathcal{T}_{L \Phi^{\dagger} \rightarrow L \Phi^{\dagger}, L^{C} \Phi}^{\mathrm{res}}\right|^{2} \\
& =\int d \operatorname{LIPS} h P_{R} S(\not p) \not p\left(h^{T} h^{*} P_{L}\right. \\
& \left.+h^{\dagger} h P_{R}\right) S^{\dagger}(\not p) P_{L} h^{\dagger} \\
& =-i h P_{R}\left[S(\not p)-S^{\dagger}(\not p)\right] P_{L} h^{\dagger} \\
& =2 h P_{R} \Im m S(\not p) P_{L} h^{\dagger},
\end{aligned}
$$

and for the CP-conjugate total rate,

$$
\begin{aligned}
\int d \operatorname{LIPS}\left|\mathcal{T}_{L^{C} \Phi \rightarrow L \Phi^{\dagger}, L^{C} \Phi}^{\mathrm{res}}\right|^{2} \\
=2 h^{*} P_{L} \Im m \bar{S}(\not p) P_{R} h^{T} \\
=2 h P_{R} \Im m S(\not p) P_{L} h^{\dagger}
\end{aligned}
$$

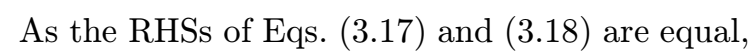
the equality $\left({ }^{\prime} . \overline{1} \overline{6}\right)$ is then obvious. Subtracting Eq. (3.13i) from Eq. (3.10 cult to see that $\Delta_{\mathrm{CP}}$ vanishes at the one-loop resummed level. We should remark that unlike [27in, the resummation approach under consideration [1] 15 - 5 satisfies CPT and unitarity exactly without recourse to any re-expansion of the resummed propagator. If we also include resummed amplitudes subleading in the Yukawa couplings, then residual $\mathrm{CP}$-violating terms which are formally of order $h_{l i}^{8}$ and higher occur in $\Delta_{\mathrm{CP}}$. These terms result from the interference of two resummed amplitudes containing one-loop vertex graphs. Because of unitarity however, the residual CP-violating terms of order $h_{l i}^{8}$ and $h_{l i}^{10}$ will cancel at two loops together with respective $\mathrm{CP}$-violating terms coming from one-loop $2 \rightarrow 4$ scatterings, and so on.

We close this section by making a few important comments. In the approach considered 
here [115], the physical transition amplitude is obtained by sandwiching the resummed propagators between matrix elements related to initial and final states of the resonant process. Therefore, diagonalization of $S(\not p)$ is no more necessary, thereby avoiding possible singularities emanating from non-diagonalizable (Jordan-like) effective Hamiltonians [or equivalently $\left.S^{-1}(p)\right]$ [ [1 $155_{1}$. In fact, such effective Hamiltonians represent situations in which the $\mathrm{CP}$-violating mixing between the two unstable particles reaches its maximum and physical CP asymmetries are therefore large. In such a case, the complex mass eigenvalues of the effective Hamiltonian are exactly equal. How can then this observation be reconciled with the CP-invariance condition (2.2i)? To answer this question, we should notice that in the presence of a large particle mixing, the mass eigenstates of $S^{-1}(\not p)$ are generally non-unitary among themselves, whereas the OS-renormalized mass eigenstates [205] form a well-defined unitary basis (or any other renormalization scheme that preserves orthonormality of the Hilbert space), upon which perturbation theory can be formulated order by order. Therefore, the field-theoretic OS renormalized masses are those that enter the condition of CP invariance given in Eq. (2.2i). Consequently, if the two complex mass eigenvalues of the effective Hamiltonian are equal, this does not necessarily entail an equality between their respective OS renormalized masses, and, hence, absence of $\mathrm{CP}$ violation as well [i1 $\left.1 \overline{5}_{1}^{n}\right]$.

\section{Low-energy constraints}

It is interesting to look briefly at the possible phenomenological implications of the leptogenesis model for collider $[2 \overline{8} \bar{i}$ and lower energies. However, if the out-of-equilibrium constraints are imposed on all lepton families of the model, all possible new-physics phenomena are estimated to be extremely small at the tree or one-loop level. Since sphaleron interactions preserve the individual quantum numbers $B / 3-L_{i}$, e.g., $B / 3-$ $L_{e}$, it is sufficient to produce the observed asymmetry in $B$ by an excess in $L_{e}$. Most interestingly, the so-generated BAU will not be washed out, even if operators that violate $L_{\mu}$ and $L_{\tau}$ are in thermal equilibrium provided that possible

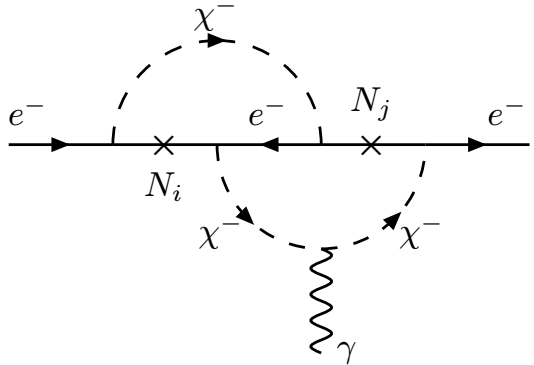

Figure 3: Typical two-loop diagram contributing to the EDM of electron.

$L_{e}-L_{\mu^{-}}$and $L_{e}-L_{\tau^{-}}$violating interactions are absent $\left[\overline{2} \overline{9}_{i}^{\prime}\right.$. This is not very hard to imagine. For example, one may have two right-handed neutrinos that mix with the electron family only and produce the $\mathrm{BAU}$, and the remaining isosinglet neutrinos strongly mix with the $\mu$ and $\tau$ families. This class of heavy Majorana neutrino models may predict sizeable new-physics phenomena due to significant non-decoupling quantum effects on lepton-flavour-violating decays of the $Z$ boson

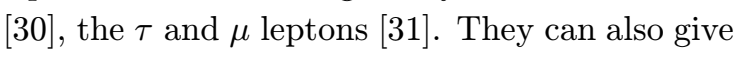
rise to breaking of universality in leptonic diagonal $Z$-boson [32] and $\pi$ decays [2 $25 \bar{n}$, etc. [33in].

Below the critical temperature $T_{c}$ of the firstorder electroweak phase transition, the Higgs boson acquires a non-vanishing vacuum expectation value $v$ which leads to non-zero light neutrino masses. However, for the models under discussion, light-neutrino masses scale as $m_{\nu} \sim$ $\left(v^{2} \mu_{L}\right) / M^{2}$, and therefore satisfy easily the cosmological upper bound of $10 \mathrm{eV}$, even if $M$ is as low as $1 \mathrm{TeV}$.

The leptogenesis model gives rise to an EDM of electron at two loops [34], on which the tight experimental bound $\left(d_{e} / e\right)<10^{-26} \mathrm{~cm}\left[35^{5}\right]$ applies. A typical diagram is shown in Fig. 3. A first estimate for $m_{N_{2}}, m_{N_{1}} \gg M_{W}$ yields

$$
\begin{aligned}
\left(\frac{d_{e}}{e}\right) & \sim\left(10^{-24} \mathrm{~cm}\right) \Im m\left(h_{1 e} h_{2 e}^{*}\right)^{2} \\
& \times \frac{m_{N_{1}} m_{N_{2}}\left(m_{N_{1}}^{2}-m_{N_{2}}^{2}\right)}{\left(m_{N_{1}}^{2}+m_{N_{2}}^{2}\right)^{2}} \ln \left(\frac{m_{N_{1}}}{M_{W}}\right),
\end{aligned}
$$

The above EDM contribution is several orders of magnitude below the experimental bound, if 
$x_{N}=\left(m_{N_{2}} / m_{N_{1}}\right)-1<10^{-3}$ or $\left|h_{l 1}\right|,\left|h_{l 2}\right| \lesssim$ $10^{-2}$.

\section{Finite temperature effects and Boltzmann equations}

In the leptogenesis scenario based on mixing of heavy neutrinos, one has to worry about other effects, which might, to some extend, affect the resonant condition of $\mathrm{CP}$ violation in Eq. (3.9). Apart from the intrinsic width of a particle resonance, there may be broadening effects at high temperatures due to collisions among particles. Such effects will contribute terms of order $h_{l i}^{4}$ to the $N_{i}$ widths and are small in general [36]. the other hand, finite temperature effects on the $T=0$ masses of particles may be significant. Because of the SM gauge interactions, the leptons and the Higgs fields receive appreciable thermal masses to [3근, i.e.

$$
\frac{m_{L}^{2}(T)}{T^{2}}=\frac{1}{32}\left(3 g^{2}+g^{2}\right) \approx 0.044
$$

where $g$ and $g^{\prime}$ are the $\mathrm{SU}(2)_{L}$ and $\mathrm{U}(1)_{Y}$ gauge couplings at the running scale $M_{Z}$. The isosinglet heavy neutrinos also acquire thermal masses through Yukawa interactions [37'], i.e.

$$
\frac{m_{N_{i}}^{2}(T)-m_{N_{i}}^{2}(0)}{T^{2}}=\frac{1}{16}\left|h_{l i}\right|^{2} .
$$

Such a $T$-dependent mass shift is small and comparable to the $N_{i}$ widths at $T \approx m_{N_{i}}$. Therefore, it is easy to see that the condition for resonant $\mathrm{CP}$ violation through mixing in Eq. (3.9.) is qualitatively satisfied. Finally, the Higgs field also receives appreciable thermal contributions. The authors in [38'] have calculated the one-loop Higgs thermal mass. They find that $M_{\Phi}(T) / T \lesssim$ 0.6 for LEP2 favoured values of the Higgs-boson mass, i.e., $M_{H}<200$. In this Higgs-mass range, the effective decay widths of the heavy neutrinos $\Gamma_{N_{i}}(T)$ will be reduced relative to $\Gamma_{N_{i}}(0)$ by a factor of two or three due to sizeable phase-space corrections. Nevertheless, the influence on the resonant phenomenon of $\mathrm{CP}$ violation through mixing is not dramatic when the latter effects are included, and therefore large leptonic CP asymmetries can still be generated.
Before we present plots of numerical estimates, it is useful to discuss first the out-of equilibrium constraints on heavy neutrino decays [3 39 '] These constraints are qualitatively given by

$$
\Gamma_{N_{i}}\left(T=m_{N_{i}}\right) \lesssim 2 K H\left(T=m_{N_{i}}\right)
$$

where $K \approx 1-1000$ is a factor that quantifies how far from the expansion rate of Universe heavyneutrino lifetimes are and $H(T)$ is the Hubble parameter

$$
H(T)=1.73 g_{*}^{1 / 2} \frac{T^{2}}{M_{\text {Planck }}},
$$

with $M_{\text {Planck }}=1.210^{19} \mathrm{GeV}$ and $g_{*} \approx 100-400$ being the number of active degrees of freedom in usual extensions of the SM. Then, the out-of equilibrium constraint in Eq. (5.3i) translates into the bound

$$
\left|h_{l i}\right|^{2} \lesssim 7.2 K \times 10^{-14}\left(\frac{m_{N_{i}}}{1 \mathrm{TeV}}\right)
$$

The above bound is considered for all Yukawa couplings.

Above the electroweak phase transition, the $B+L$-sphaleron interactions convert approximately one-third of the lepton-to-entropy density ratio $Y_{L}=n_{L} / s$ into a baryon-to-entropy density ratio $Y_{B}=n_{B} / s$, i.e. [[4]

$$
Y_{B} \approx-\frac{1}{3} Y_{L} \approx-\frac{1}{3 K} \frac{\delta_{N_{i}}}{g_{*}} .
$$

The last approximate equality represents the asymptotic solution of the relevant Boltzmann equa-

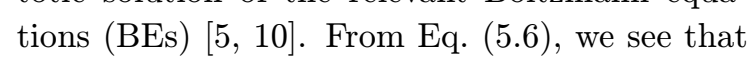
$Y_{B}$ can be in observed ball park, i.e. $Y_{B} \approx 10^{-10}$, if $\left|\delta_{N_{i}}\right| / K$ are of order $10^{-7}-10^{-6}$. Clearly, $\mathrm{CP}$ asymmetries of order unity allow for very large values of $K$. As a consequence, the thermal plasma can then be rather dense and the conditions of kinetic equilibrium in BEs can comfortably be satisfied even within the minimal leptogenesis scenario.

In Fig. 4, we plot the dependence of $Y_{L}(x)$ on $x=m_{N_{1}} / T$ for two representative scenarios, after solving numerically the BEs [110']. The observed range for $Y_{L}, Y_{L}^{o b s}$, is indicated with two confining horizontal dotted lines. The parameter $x_{N}=m_{N_{2}} / m_{N_{1}}-1$ is a measure of the degree of mass degeneracy for $N_{1}$ and $N_{2}$. In 

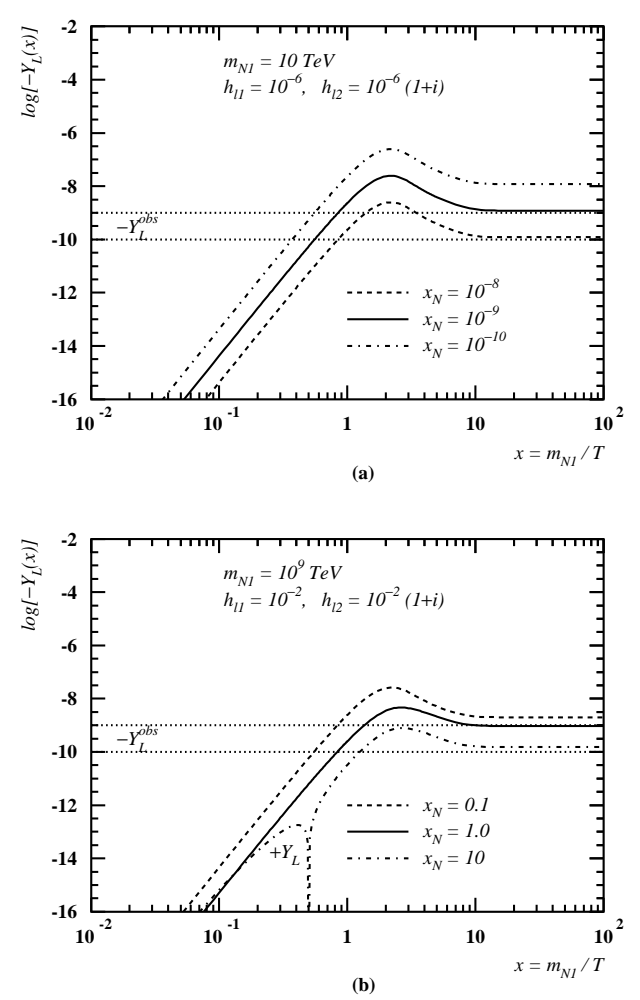

Figure 4: Lepton asymmetries for selected heavy Majorana neutrino scenarios.

the first scenario in Fig. 4(a), $m_{N_{2}} \geq m_{N_{1}}=10$ $\mathrm{TeV}$, and $h_{l 1}=10^{-6}, h_{l 2}=10^{-6}(1+i)$. In this case, a heavy-neutrino mass splitting $x_{N}$ of order $10^{-9}-10^{-8}$ is sufficient to account for the BAU. We also find that the $\varepsilon$-type $\mathrm{CP}$-violating mechanism is dominant, whereas $\varepsilon^{\prime}$-type $\mathrm{CP}$ violation is extremely suppressed. The second scenario in Fig. 4(b) is close to the traditional one considered in [i], where $m_{N_{1}}=10^{9} \mathrm{TeV}$, and $h_{l 1}=10^{-2}$ and $h_{l 2}=10^{-2}(1+i)$. Obviously, it is not mandatory here to have a high degree of degeneracy for $N_{1}$ and $N_{2}$ in order to get sufficient CP violation for the BAU. In this case, both $\varepsilon$ - and $\varepsilon^{\prime}$ - type mechanisms of $\mathrm{CP}$ violation are equally important.

\section{Conclusions}

We have considered the scenario of baryogenesis through leptogenesis in which out-of-equilibrium
$L$-violating decays of heavy Majorana neutrinos produce an excess in $L$, which is converted into the observed asymmetry in $B$, through the $B+L$ violating sphaleron interactions. In particular, we have studied the impact of the $\varepsilon$ - and $\varepsilon^{\prime}$-type mechanisms for $\mathrm{CP}$ violation on generating the required excess of baryons detected in the Universe. Based on the effective resummation approach outlined in Section 3, we have found that $\varepsilon$-type $\mathrm{CP}$ violation is resonantly enhanced if the mass splitting of the heavy Majorana neutrinos is comparable to their widths (c.f. Eq. ( $\overline{3} . \overline{9})$ ), and if the parameter $\delta_{C P}$ defined in Eq. (i. a value close to one. These are the two necessary and sufficient conditions for resonant $\mathrm{CP}$ violation of order unity [1 100.1 . As a consequence, the scale of lepogenesis may be lowered up to the $\mathrm{TeV}$ range. In fact, $\mathrm{E}_{6}$-motivated scenarios with nearly degenerate heavy Majorana neutrinos and all Yukawa couplings being of the same order can still be responsible for the BAU. In this kinematic range, the $\varepsilon^{\prime}$-type contributions are extremely suppressed. Even though interesting alternatives have been proposed in the literature [ [411] , the leptogenesis model considered here is the most economical one. Just two isosinglet neutrinos are needed for baryogenesis. Finally, we must remark that finite temparature effects and constraints due to electron EDM have a rather marginal effect on $\mathrm{CP}$ asymmetries.

\section{References}

[1] M. Fukugita and T. Yanagida, Phys. Lett. B174 (1986) 45.

[2] V.A. Kuzmin, V.A. Rubakov and M.E. Shaposhnikov, Phys. Lett. B155 (1985) 36.

[3] P. Arnold and L. McLerran, Phys. Rev. D36 (1987) 581; D37 (1988) 1020.

[4] A. Bochkarev and M.E. Shaposhnikov, Mod. Phys. Lett. A2 (1987) 417; Mod. Phys. Lett. A2 (1987) 921; S. Yu. Khlebnikov and M.E. Shaposhnikov, Nucl. Phys. B308 (1988) 885; E. Mottola and A. Wipf, Phys. Rev. D39 (1989) 588.

[5] M.A. Luty, Phys. Rev. D45 (1992) 455.

[6] C.E. Vayonakis, Phys. Lett. B286 (1992) 92.

[7] P. Langacker, R.D. Peccei and T. Yanagida, Mod. Phys. Lett. A1 (1986) 541; R.N. Mohapa- 
tra and X. Zhang, Phys. Rev. D45 (1992) 5331; K. Enqvist and I. Vilja, Phys. Lett. B299 (1993) 281; A. Acker, H. Kikuchi, E. Ma and U. Sarkar, Phys. Rev. D48 (1993) 5006; P.J. O'Donnell and U. Sarkar, Phys. Rev. D49 (1994) 2118; M.P. Worah, Phys. Rev. D53 (1996) 3902; M. Plümacher, Z. Phys. C74 (1997) 549; L. Covi, E. Roulet and F. Vissani, Phys. Lett. B384 (1996) 169; W. Buchmüller and M. Plümacher, Phys. Lett. B389 (1996) 73.

[8] J. Liu and G. Segré, Phys. Rev. D48 (1993) 4609.

[9] M. Flanz, E.A. Paschos, U. Sarkar and J. Weiss, Phys. Lett. B389 (1996) 693; L. Covi and E. Roulet, Phys. Lett. B399 (1997) 113; E.Kh. Akhmedov, V.A. Rubakov and A.Yu. Smirnov, hep-ph/9803255.

[10] A. Pilaftsis, Phys. Rev. D56 (1997) 5431.

[11] For a review, see, E.A. Paschos and U. Türke, Phys. Rep. 178 (1989) 147; P.K. Kabir, "The CP puzzle," Academic Press, London and New York, 1968.

[12] A. Yu. Ignatiev, V.A. Kuzmin and M.E. Shaposhnikov, JETP Lett. 30 (1979) 688; F.J. Botella and J. Roldan, Phys. Rev. D44 (1991) 966.

[13] J. Papavassiliou and A. Pilaftsis, Phys. Rev. Lett. 75 (1995) 3060; Phys. Rev. D53 (1996) 2128; Phys. Rev. D54 (1996) 5315.

[14] A. Pilaftsis, Phys. Rev. Lett. 77 (1996) 4996.

[15] A. Pilaftsis, Nucl. Phys. B504 (1997) 61.

[16] A. Pilaftsis, Z. Phys. C47 (1990) 95; A. Pilaftsis and M. Nowakowski, Phys. Lett. B245 (1990) 185; Mod. Phys. Lett. A6 (1991) 1933.

[17] H. Fritzsch and P. Minkowski, Ann. Phys. (N.Y.) 93 (1975) 193.

[18] D. Wyler and L. Wolfenstein, Nucl. Phys. B218 (1983) 205.

[19] E. Witten, Nucl. Phys. B268 (1986) 79; R.N. Mohapatra and J.W.F. Valle, Phys. Rev. D34 (1986) 1642; S. Nandi and U. Sarkar, Phys. Rev. Lett. 56 (1986) 564; J.W.F. Valle, Prog. Part. Nucl. Phys. 26 (1991) 91.

[20] J. Bernabéu, G.C. Branco and M. Gronau, Phys. Lett. B169 (1986) 243.

[21] J.G. Körner, A. Pilaftsis and K. Schilcher, Phys. Rev. D47 (1993) 1080.

[22] L. Stodolsky, Phys. Rev. D36 (1987) 2273.
[23] M. Veltman, Physica 29 (1963) 186.

[24] H. Lehmann, K. Symanzik, and W. Zimmermann, Nuovo Cim. 1 (1955) 439; N. Bogoliubov and D. Shirkov, Fortschr. der Phys. 3 (1955) 439; N. Bogoliubov, B. Medvedev, and M. Polivanov, Fortschr. der Phys. 3 (1958) 169.

[25] B.A. Kniehl and A. Pilaftsis, Nucl. Phys. B474 (1996) 286.

[26] E. Roulet, L. Covi and F. Vissani, Phys. Lett. B424 (1998) 101; M. Flanz and E.A. Paschos, hep-ph/9805427.

[27] W. Buchmüller and M. Plümacher, hepph/9710460 (revised on 4 May 1998).

[28] For like-sign dilepton signals due to heavy Majorana neutrinos at the CERN Large Hadron Collider (LHC), see, A. Datta, M. Guchait and A. Pilaftsis, Phys. Rev. D50 (1994) 3195.

[29] B.A. Campbell, S. Davidson, J. Ellis and K.A. Olive, Phys. Lett. B297 (1992) 118; H. Dreiner and G.G. Ross, Nucl. Phys. B410 (1993) 188.

[30] J.G. Körner, A. Pilaftsis and K. Schilcher, Phys. Lett. B300 (1993) 381.

[31] A. Pilaftsis, Mod. Phys. Lett. A9 (1994) 3595; M.C. Gonzalez-Garcia and J.W.F. Valle, Mod. Phys. Lett. A7 (1992) 477; E9 (1994) 2569; A. Ilakovac and A. Pilaftsis, Nucl. Phys. B437 (1995) 491; G. Bhattacharya, P. Kalyniak and I. Mello, Phys. Rev. D51 (1995) 3569; A. Ilakovac, B.A. Kniehl, and A. Pilaftsis, Phys. Rev. D52 (1995) 3993; P. Kalyniak and I. Mello, Phys. Rev. D55 (1997) 1453.

[32] J. Bernabéu, J.G. Körner, A. Pilaftsis and K. Schilcher, Phys. Rev. Lett. 71 (1993) 2695; J. Bernabéu and A. Pilaftsis, Phys. Lett. B351 (1995) 235.

[33] For a review, see, P. Langacker and D. London, Phys. Rev. D38 (1988) 886.

[34] D. Ng and J.N. Ng, Mod. Phys. Lett. A11 (1996) 211.

[35] Particle Data Group, R.M. Barnett et al., Phys. Rev. D54 (1996) 1.

[36] L. Covi, N. Rius, E. Roulet and F. Vissani, hep$\mathrm{ph} / 9704366$.

[37] H.A. Weldon, Phys. Rev. D26 (1982) 2789.

[38] J.M. Cline, K. Kainulainen and K.A. Olive, Phys. Rev. D49 (1994) 6394.

[39] E.W. Kolb and M.S. Turner, The Early Universe, (Addison-Wesley, Reading, MA, 1989). 
[40] J.A. Harvey and M.S. Turner, Phys. Rev. D42 (1990) 3344.

[41] E. Ma and U. Sarkar, Phys. Rev. Lett. 80 (1998) 5716; hep-ph/9807307. 